\title{
Men's Participation and Performance in the Boston Marathon from 1897 to 2017
}

\author{
Authors \\ Beat Knechtle1, Stefania Di Gangi², Christoph Alexander Rüst ${ }^{2}$, Thomas Rosemann ${ }^{3}$, Pantelis Theodoros Nikolaidis ${ }^{4}$
}

\section{Affiliations}

1 Gesundheitszentrum, St. Gallen, St. Gallen, Switzerland

2 Institute of Primary Care, University of Zurich, Zurich, Switzerland

3 Department of General Practice, University of Zurich, Zurich, Switzerland

4 Exercise Physiology Laboratory, Nikaia, Exercise Physiology, Nikaia, Greece

\section{Key words}

cardiorespiratory fitness, endurance, men, history, running, performance

accepted 06.07.2018

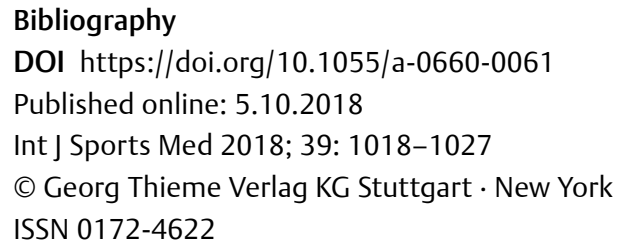

\section{Correspondence}

Dr. Beat Knechtle

Gesundheitszentrum

St. Gallen

Vadianstr. 26

St. Gallen

9001

Switzerland

Tel.: + 41/71/226 82 82, Fax: +41/71/226 8272

beat.knechtle@hispeed.ch

\begin{abstract}
This study examined men's participation and performance in the Boston Marathon. Data from all 368,940 official male finishers in the Boston Marathon from 1897 through 2017 were analyzed using different analyses and regression models for all runners, annual top hundred finishers, annual top ten finishers and annual winners. Effect of calendar year on race time was examined alone, in a uni-variable model, and together with country of origin and weather conditions (average air temperature and precipitation) in a multi-variable model. The mean race time of all finishers increased across calendar years, in line with the participation, but it decreased when we considered the annual winners, 10 and 100 fastest. Kenyans and Ethiopians were the fastest nationalities $(p<0.001)$ when we considered all finishers and the annual top 100 fastest but not when the annual 10 fastest were considered. Air temperature $\leq 8{ }^{\circ} \mathrm{C}$ improved $(\mathrm{p}<0.001)$ race times compared to air temperature $>8^{\circ} \mathrm{C}$. Precipitation ( $>0 \mathrm{~mm}$ ) improved performance for the annual 100 fastest and annual 10 fastest ( $p=0.013-0.031$ ) but not for all finishers. Our findings improved the knowledge about the evolution of male marathoners across calendar years, considering as main effects country of origin and particular weather conditions.
\end{abstract}

\section{Introduction}

Among the road-based running races, marathon running is of high popularity. For example, the number of overall finishers in marathon races held in the United States of America was 507,600 in 2016, slightly lower than in 2015 with 509,000 finishers. A total of 1,100 marathons were held in both 2016 and 2015 (www.runningusa.org/marathon-report-2017).

Marathon running for men has been part of the Olympic Games since 1896 (www.sports-reference.com/olympics/summer/1896/ ATH/mens-marathon.html). The oldest non-Olympic annual mar- athon race is the Boston Marathon, held continuously since 1897 (www.baa.org/races/boston-marathon/boston-marathon-history. aspx). To date, we know a bit about trends in marathon running after 1976 [2], but we have no knowledge about trends in participation in marathon running since the beginning of large city marathons such as the Boston Marathon or the New York City Marathon. We know that the participation of age group marathoners in large city marathons such as the New York City Marathon increased at the end of the last century $[8,12]$ and the number of women increased after 1972 when the first women were allowed to officially 
run in large city marathons $[10,19]$. Moreover, master athletes of very high ages older than 75 years continue increasing their participation and improving their performance [1].

Apart from participation, the trend in performance in marathon running is also of interest. Current studies have mainly investigated performance trends in elite [13] and recreational age groups [27] competing in recent years or decades in large city marathons. However, we have no knowledge about the performance trends in elite and recreational athletes since the beginning of marathon running in 1897. It would be interesting to see both annual data on men's participation and men's average race times. This would provide both cultural and scientific information to historians and sports scientists alike.

A further aspect is the nationality of the marathoners. It is well known that East African runners from Ethiopia and Kenya are the fastest in marathon running when the fastest race times in marathon running [17] and the races of the World Marathon Majors [9] were analyzed. We have, however, no knowledge about the trends in participation and performance of East African marathoners competing in large city marathons. Particularly, we do not know for specifically when East African marathoners entered the large city marathons. For the Boston Marathon, Kenya's Ibrahim Hussein finished in 1988 one second ahead of Tanzania's Juma Ikangaa, and became the first African to win the Boston Marathon, or any other major marathon (www.baa.org/races/boston-marathon/boston-marathon-history/boston-marathon-milestones.aspx).

The Boston Marathon is always held on Patriots' Day, the third Monday of April. The race is a point-to-point course from east to west with a big weather influence [13], where warmer temperatures and headwinds on the day of the race slow winning times [15]. For a fast time in the Boston Marathon, the temperature should be below $8{ }^{\circ} \mathrm{C}[23]$. When the runners have a tailwind, they get a 'push' for $42 \mathrm{~km}$. This might explain why Geoffrey Mutai from Kenya set in 2011 a new course record as well as a new world's best time of 2:03:02 h:min:sec and the top four men all finished under the old course record. The wind was from WSW (West-South-West) with a speed of $15 \mathrm{mph}(24.14 \mathrm{~km} / \mathrm{h})$ with a maximal wind speed of $26 \mathrm{mph}(41.84 \mathrm{~km} / \mathrm{h})$, and a maximal gust speed of $35 \mathrm{mph}$ $(56.32 \mathrm{~km} / \mathrm{h})$.

The aim of the present study was, therefore, to investigate participation and performance trends in male marathoners competing between 1897 and 2017 in the Boston Marathon with the hypothesis that participation increased and performance improved in the last 120 years in this city marathon. Regarding nationality, we hypothesized that East African runners would enter the race in the seventies and be the fastest from their entry in the race to the present. Since race times in Boston Marathon are highly influenced by environmental conditions (i. e. temperature, wind direction, and wind speed), we also considered these characteristics in our analyses.

\section{Materials and Methods}

\section{Ethical approval}

This study was approved by the Institutional Review Board of Kanton St. Gallen, Switzerland, with a waiver of the requirement for informed consent of the participants as the study involved the anal- ysis of publicly available data. The research was conducted ethically according to international standards and as described by Harriss and colleagues [7].

\section{Data sampling and data analysis}

The Boston Marathon is the world's oldest annual city marathon (www.baa.org/races/boston-marathon/boston-marathon-history. aspx). To compete in this race, athletes must meet time standards that correspond to age and sex and that have changed over years (www.baa.org/races/boston-marathon/participant-information/ qualifying.aspx).

Data for the 120 years of competition from 1897 to 2017 were obtained from the official race website (www.baa.org/races/boston-marathon.aspx). Available information was name, surname, nationality of runners, sex and runners' year of competition, and race times. We integrated this dataset with other information on year 2005 . For the year 2005, race results from the official race website were incomplete, and we obtained them from the website www.marathonguide.com/results.

To identify observations from a single runner, we defined an id variable with name, surname, nationality and period of competition, supposing that a single runner could participate at most for 25 years. All male finishers in the history of the Boston Marathon ( $n=368,940)$ were analyzed.

Since temperature and wind including direction seemed to have an influence on race time in the Boston Marathon [13, 15, 23], we merged the database with additional information on the weather conditions during the days of the race: average temperature, precipitation in mm, humidity level, wind direction and wind speed. Weather data (i. e. temperatures, precipitation, humidity, wind direction, wind speed) were obtained from different websites (http:// w2.weather.gov/climate/local_data.php?wfo=box; www.baa.org/ races/boston-marathon/boston-marathon-history/weather-conditions.aspx; http://findmymarathon.com/weather-detail. php?zname $=$ Boston \%20Marathon\&year $=$; www.wunderground . com/history/airport/KBOS/2013/1/15/MonthlyHistory.html).

Data about temperature and precipitation were available for the entire 120 years. Other information (i.e. humidity, wind direction and wind speed) was available from 1945 onward.

\section{Statistical analysis}

All data are presented as means \pm standard deviation for continuous variables and as number $\mathrm{N}$ (\%) for categorical variables. Calendar year was considered as both a discrete value of a continuous variable and as an endpoint of a time-interval categorical variable. In fact, to compare performance by period of time, we grouped each calendar year into time-periods of 20 years. We used the following conventions: [year1, year2) denoted interval from year 1 (included) to year2 (excluded) and [year1, year2] denoted interval from year1 (included) to year2 (included). Performance, or race time, was recorded in the format "hours:minutes:seconds". The acceptable type I error was set at $p \leq 0.05$. All statistical analyses were carried out using statistical package R, R Core Team (2016). R: A language and environment for statistical computing. R Foundation for Statistical Computing, Vienna, Austria. URL https:// www.R-project.org/. 
For data visualization, we used the ggplot2 package. One-way analysis of variance examined the effect of calendar year, grouped into time-periods, on race time, and the effect of country, grouped into eight geographical areas (i. e. Kenya-Ethiopia, rest of Africa, Asia, Canada, Central-South America, Europe, Oceania and USA). T-tests were performed to compare the average race time between groups of weather conditions, defined as dichotomous variables. Moreover, effect of calendar year on race time was examined alone, in a uni-variable model, and together with country of origin and weather conditions in a multi-variable model. We used a spline regression model, with a spline smooth term in function of calendar year and a linear term in function of country, temperature and precipitation. Temperature and precipitation were defined as dichotomous variables $1 / 0$ depending on, respectively, temperature below/above $8{ }^{\circ} \mathrm{C}$ and precipitation ( $>0 \mathrm{~mm} / 0 \mathrm{~mm}$ ).

Since we have repeated measurements within runners, we performed a mixed model, with random effects on intercept for each runner. We used Generalized Additive Mixed Models, gamm4 in R, which are extensions of Generalized Additive Models, allowing for repeated measurements and then for random effects. In a Generalized Additive Model, the response variable depends linearly on unknown smooth functions of some predictor variables. The univariable (1) and multi-variable (2) models were specified as follow:

(1) Race Time $(Y) \sim[$ Fixed effects $(X)=S(Y E A R, k)+[$ Random effects of intercept $=$ Runners] Race Time $(Y) \sim$ [Fixed effects $(\mathrm{X})=$ Country + Temperature $\leq 8{ }^{\circ} \mathrm{C}+$ Precipitation $>0 \mathrm{~mm}+$ $\mathrm{S}(\mathrm{YEAR}, \mathrm{k})+[$ Random effects of intercept=Runners] where $\mathrm{S}(\mathrm{YEAR}, \mathrm{k})$ is a spline, changing with calendar year and with $\mathrm{k}$ basis dimension.

(2) We performed different analyses and regression models for the following subgroups: all runners, annual top hundred finishers, annual top ten finishers and annual winners. In the multi-variable model we did not include, as predictors, other variables describing weather conditions, because we did not have enough observations to study the whole 120 -year period.

\section{Results}

\section{Participation}

Between 1897 and 2017, a total of 368,940 observations from 238,685 different finishers were recorded in the race results. Therefore we had many observations per runner. Overall, the average was 1.55 per runner, but in fact only 60,709 (25\%) runners have more than one record. These runners participated on average three times in the entire period of observation. The maximum was 26 , which means one runner participated annually during the last 25 years. As shown in $>$ Table 1, the number of finishers increased dramatically from the middle of the 1970s. Regarding country of origin, USA and their territories had the largest participation, 306,131 observations $(83 \%$ of 368,940$)$ and athletes from Kenya and Ethiopia had the smallest participation, $285(0.08 \%$ of 368,940$)$.

\section{Performance considering all finishers}

Summary statistics

In $>$ Table 1 we reported the summary statistics of the average performance overall, by time grouped into classes, by country and by weather conditions. Overall, the average race time was 03:38:21 $\pm 00: 41: 46 \mathrm{~h}: \mathrm{min}: s$. Performance had a trend to decrease over time. In the last 20 years $(1997,2017)$, race time, on average, was the slowest and in the first period of observations $(1897,1917)$ was the fastest. Exceptionally in period $(1957,1977)$, compared to the previous period, though an extremely high increase in participation, race time decreased. In \ Fig. 1, we plotted points showing the details of mean performance by calendar year. The worst overall performance was in 2004 (04:16:16 $\pm 00: 46: 53 \mathrm{~h}: \mathrm{min}: \mathrm{s})$ and the best overall performance in 1912 (02:29:31 $\pm 00: 04: 27 \mathrm{~h}: \mathrm{min}: \mathrm{s})$. It should be highlighted that there were 526 observations before 1924 , where the distance was not standardized at $42.195 \mathrm{~km} \mathrm{(26}$ miles, 385 yards). In fact, the average performance in period (1897, 1924) was $02: 51: 17 \pm 00: 18: 13 \mathrm{~h}: \mathrm{min}: \mathrm{s}$ and significantly different $(p<0.001)$ compared with the average performance of 03:02:24 $\pm 00: 18: 16 \mathrm{~h}: \mathrm{min}: \mathrm{s}$ in the rest of the period $(1924,1937)$.

Regarding the country of origin, athletes from Kenya and Ethiopia showed the fastest performance (02:18:20 $\pm 00: 12: 24 \mathrm{~h}: \mathrm{min}: \mathrm{s})$ and athletes from Europe had the slowest performance (03:46:42 \pm 00:47:00 h:min:s). We examined also the differences between single nationalities, not grouped into geographical areas, which were in total 136. We show, in Appendix, > Fig. 1S, the trend of average performance of the 49 most relevant nationalities, which had 60 or more observations each. We observed that athletes from Kenya and Ethiopia, differently from other countries, had a decreasing trend in race time. Athletes from Kenya participated for the first time in 1979 and athletes from Ethiopia in 1963.

Regarding weather conditions, we did not focus on details, as shown in $>$ Table 1 , but we found that temperatures $\leq 8^{\circ} \mathrm{C}$, precipitation $>0 \mathrm{~mm}$, wind direction from the west, wind speed $\leq 16 \mathrm{~km} / \mathrm{h}$ and humidity level $\leq 70 \%$ significantly $(p<0.001)$ improved performance.

\section{Statistical model}

In $>$ Fig. 1 and $>$ Fig. 2 we show the fitted curve, from the generalized additive mixed models, uni-variable and multi-variable, described in the methods section and whose details are reported in - Table 2. In > Fig. 2 we do not show, graphically, the effect of precipitation, but only of country and temperature. Overall, race time of all finishers increased across calendar years ( $>$ Fig. 1) in line with the participation ( $\vee$ Table $\mathbf{1}$ ).

In $>$ Table $\mathbf{2}$ we show that athletes from Kenya and Ethiopia were significantly $(p<0.001)$ the fastest, compared with every other country group. The estimates ranged from a minimum of 01:12:13 h:min:s, which was the estimated difference among Canada and Kenya-Ethiopia, and a maximum of 01:28:02 h:min:s, which was the estimated difference among Asia and Kenya-Ethiopia. Temperature level $\leq 8{ }^{\circ} \mathrm{C}$ significantly $(\mathrm{p}<0.001)$ improved performance of 00:03:35 h:min:s, compared with temperature $>8{ }^{\circ} \mathrm{C}$. Absence or presence of precipitation was not significant $(p=0.426)$.

\section{Performance considering the annual hundred fastest}

\section{Summary statistics}

A large main effect of calendar year, grouped into classes, country and weather conditions on race time was shown $(p<0.001)$ in - Table 1. In > Fig. 1, we plotted points showing the details of mean performance by calendar year. Performance overall improved, but 


\begin{tabular}{|c|c|c|c|c|c|c|c|c|c|c|c|c|c|c|c|c|c|c|c|c|c|c|c|c|c|c|c|c|c|c|c|c|c|c|c|}
\hline & 2 & 家 & & & & & & 5 & & & & & & & & & & & $\circ \mid \begin{array}{l}\tilde{O} \\
\dot{O} \\
0\end{array}$ & & & $\mid$ & & & 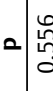 & & & 0 & & & 0 & $\mid \begin{array}{l}6 \\
0 \\
0\end{array}$ & & & \\
\hline & nُ & $\mid$ & $\begin{array}{l}\hat{\bigcap} \\
\stackrel{\leftrightarrow}{0} \\
\stackrel{0}{0}\end{array}$ & 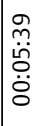 & 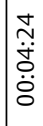 & 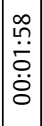 & 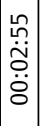 & иे & & $\begin{array}{l}\overline{\ddot{g}} \\
\ddot{\ddot{\theta}}\end{array}$ & \begin{tabular}{|l} 
o \\
$\dot{\ddot{\sigma}}$ \\
$\ddot{0}$
\end{tabular} & 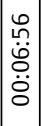 & $\begin{array}{l}\tilde{\partial} \\
\ddot{\ddot{\theta}} \\
\ddot{\theta}\end{array}$ & 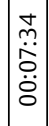 & & 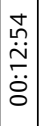 & & & $\hat{n}$ & $\begin{array}{l}\mathscr{0} \\
\ddot{\infty} \\
\dot{o} \\
\ddot{\theta}\end{array}$ & Q & 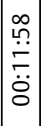 & $\mid \begin{array}{c}\overline{\hat{m}} \\
\ddot{\dot{\theta}} \\
\end{array}$ & & ڤิ) & 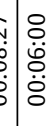 & & ज) & 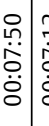 & 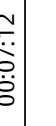 & ติ & 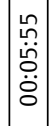 & $\begin{array}{l}0 \\
\stackrel{0}{0} \\
\stackrel{\leftrightarrow}{0} \\
\dot{8}\end{array}$ & & \\
\hline & $=$ & 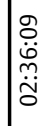 & 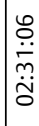 & $\begin{array}{l}\tilde{n} \\
\stackrel{\sim}{\tilde{N}} \\
\tilde{\tilde{\sigma}}\end{array}$ & 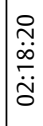 & 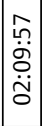 & 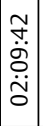 & 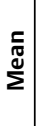 & & 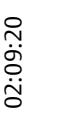 & \begin{tabular}{|l}
$\tilde{\partial}$ \\
$\ddot{\theta}$ \\
$\dot{\tilde{\sigma}}$
\end{tabular} & 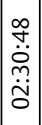 & $\begin{array}{l}\frac{a}{\ddot{n}} \\
\ddot{\tilde{\Xi}} \\
\dot{\sigma}\end{array}$ & 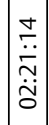 & 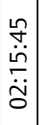 & $\mid \begin{array}{l}\tilde{c} \\
\dot{\sim} \\
\dot{\tilde{d}}\end{array}$ & & & 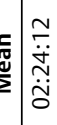 & $\mid \begin{array}{l}n \\
\stackrel{n}{\alpha} \\
\ddot{\tilde{\sigma}}\end{array}$ & $\begin{array}{l}\mathrm{c} \\
\stackrel{\Xi}{\Sigma} \\
\Sigma \\
\Sigma\end{array}$ & $\mid \begin{array}{l}\tilde{m} \\
\dot{\tilde{n}} \\
\ddot{\tilde{n}} \\
\tilde{0}\end{array}$ & $\mid \begin{array}{l}m \\
\dot{\partial} \\
\dot{\tilde{\tilde{\sigma}}} \\
0\end{array}$ & & 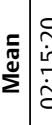 & 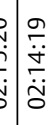 & & 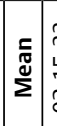 & 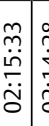 & 兑 & 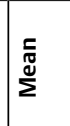 & $\mid \begin{array}{c}\overline{\tilde{r}} \\
\dot{\tilde{m}} \\
\ddot{\tilde{\sigma}}\end{array}$ & 总 & & \\
\hline & ๑ & $\begin{array}{l}\hat{n} \\
\underline{\omega} \\
\underline{6}\end{array}$ & $\mid \begin{array}{l}\hat{n} \\
\underline{\omega}\end{array}$ & $\begin{array}{l}\hat{n} \\
\underline{\omega}\end{array}$ & $\begin{array}{l}\hat{n} \\
\underline{\varrho} \\
\underline{n}\end{array}$ & $\mid \begin{array}{l}n \\
\hat{6} \\
\subseteq\end{array}$ & $\mid \begin{array}{l}\stackrel{0}{2} \\
? \\
\sim \\
-\end{array}$ & $\circ \circ$ & & $\begin{array}{l}m \\
\infty \\
\stackrel{\sim}{0}\end{array}$ & $\stackrel{\curvearrowright}{\sigma}$ & $\mid \begin{array}{l}\stackrel{0}{\mathfrak{n}} \\
\stackrel{1}{\simeq}\end{array}$ & 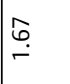 & $\mid \begin{array}{c}m \\
m \\
m \\
m\end{array}$ & $\begin{array}{c}\tilde{m} \\
0 \\
0 \\
0\end{array}$ & $\begin{array}{l}\hat{b} \\
\dot{\sigma}\end{array}$ & & & 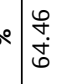 & 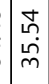 & & $\mid \begin{array}{l}\hat{0} \\
\dot{\sigma} \\
\end{array}$ & $\left|\begin{array}{c}m \\
m \\
\infty \\
m\end{array}\right|$ & & $\circ$ & $\begin{array}{r}: \\
\vdots \\
\vdots\end{array}$ & & $\circ$ & 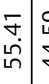 & 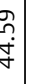 & $\propto^{\circ}$ & $\mid \begin{array}{c}\tilde{w} \\
\dot{m} \\
\dot{m}\end{array}$ & 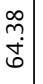 & & \\
\hline & $\tilde{I}$ & iे & $\stackrel{\sim}{\sim}$ & $\stackrel{\sim}{\sim}$ & $\stackrel{\sim}{\sim}$ & $\stackrel{\sim}{*}$ & $\bar{\sim}$ & $z$ & & $\stackrel{\Perp}{\sim}$ & $=$ & $\stackrel{\Perp}{\sim}$ & $\sim$ & $\div$ & - & in & - & $z$ & $z \stackrel{\infty}{\wedge}$ & $q$ & $z$ & 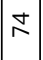 & $\mathscr{q}$ & -2 & $z$ & $i$ & 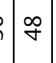 & $z$ & $\bar{\gamma}$ & $m \mid F$ & $z$ & $\stackrel{\circ}{\sim}$ & $f$ & $\stackrel{\infty}{+}$ & \\
\hline & 2 & $\begin{array}{l}\bar{\delta} \\
\bar{v} \\
\bar{v}\end{array}$ & & & & & & $=$ & & & & & & & & & & & $\circ \mid \begin{array}{l}\bar{\delta} \\
\dot{v} \\
\dot{v}\end{array}$ & & & $\mid \begin{array}{l}\bar{b} \\
\dot{v} \\
\dot{v}\end{array}$ & & & $=\mid \begin{array}{l}a \\
\vdots\end{array}$ & & & & 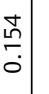 & & 0 & $\mid \begin{array}{l}\bar{\sigma} \\
\dot{d} \\
\dot{v}\end{array}$ & & & \\
\hline & 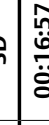 & $\frac{n}{\ddot{\theta}}$ & 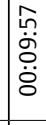 & 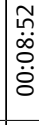 & 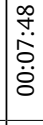 & 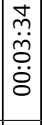 & $\mid \begin{array}{l}\infty \\
0 \\
\dot{0} \\
0 \\
0 \\
0\end{array}$ & ทे & 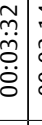 & 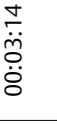 & \begin{tabular}{|l}
$\stackrel{N}{N}$ \\
$\ddot{\ddot{\theta}}$ \\
$\ddot{\ddot{\theta}}$
\end{tabular} & \begin{tabular}{c}
0 \\
0 \\
\hdashline \\
$\mid \begin{array} { c } { 0 } \\
{ 0 } \\
{ \hdashline } \\
{ }$
\end{tabular} \\
\end{tabular}$} &{\begin{array}{|l|} \\
\vdots \\
0 \\
0 \\
\ddot{O}\end{array}} &{\mid \begin{array}{l}o \\
\dot{0} \\
\dot{0} \\
\dot{0}\end{array}} &{\text { 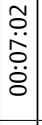 }} &{\text { 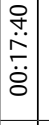 }} &{ } &{ } &{\hat{n}} &{\text { 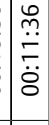 }} &{\text { जे }} &{\mid \begin{array}{c}\hat{m} \\
\dot{\omega} \\
\ddot{\ddot{o}}\end{array}} &{\text { 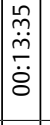 }} &{ } &{\text { ڤิ) }} &{\text { 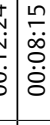 }} &{ } &{\text { ติ }} &{\begin{array}{l}0 \\
\vdots \\
\dot{0} \\
\ddot{0}\end{array}} &{ } &{\text { iे }} &{\mid \begin{array}{l}n \\
\tilde{\Xi} \\
\vdots \\
\dot{0} \\
\end{array}} &{\text { 学 }} &{ } &{ } \\
{\hline} &{\text { 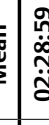 }} &{\text { 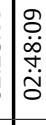 }} &{\text { 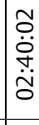 }} &{\text { 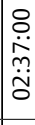 }} &{\begin{array}{|l}\stackrel{m}{\ddot{\sim}} \\
\ddot{\tilde{d}} \\
\end{array}} &{\text { 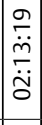 }} &{\mid \begin{array}{l}\tilde{\sim} \\
\tilde{\tilde{T}} \\
\ddot{\tilde{\tilde{U}}} \\
\end{array}} &{\sum_{\substack{\tilde{c} \\
\Sigma}}} &{\text { 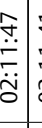 }} &{\text { 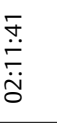 }} &{\text { 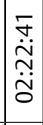 }} &{\text { 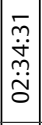 }} &{\mid \begin{array}{l}\stackrel{\infty}{0} \\
\ddot{\infty} \\
\ddot{\ddot{\alpha}} \\
\end{array}} &{\mid \begin{array}{l}\hat{0} \\
\dot{\check{n}} \\
\ddot{\tilde{d}}\end{array}} &{\text { 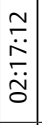 }} &{\begin{array}{l}0 \\
\dot{\tilde{\omega}} \\
\dddot{\tilde{U}} \\
0\end{array}} &{ } &{ } &{\text { 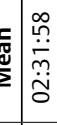 }} &{\begin{array}{l}\tilde{\tilde{N}} \\
\stackrel{\tilde{N}}{\tilde{\tilde{\sigma}}}\end{array}} &{\mid \begin{array}{c}\frac{c}{\tilde{n}} \\
\Sigma \\
\Sigma\end{array}} &{\begin{array}{l}\vdots \\
\dot{\bar{m}} \\
\dot{\tilde{g}} \\
\dot{0}\end{array}} &{\text { 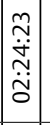 }} &{ } &{\text { 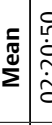 }} &{\text { 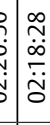 }} &{ } &{\text { 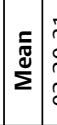 }} &{ } &{ } &{\mid \begin{array}{l}\bar{\Sigma} \\
\text { 苋 }\end{array}} &{\text { 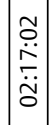 }} &{\text { 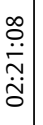 }} &{ } &{ } \\
{\hline} &{\text { ๑ }} &{\begin{array}{l}\hat{m} \\
\underline{\omega}\end{array}} &{\underset{f}{\tilde{\omega}}} &{\begin{array}{l}\stackrel{\leftrightarrow}{0} \\
\dot{\leftrightarrow}\end{array}} &{\begin{array}{l}\stackrel{0}{0} \\
\dot{0} \\
\end{array}} &{\mid \begin{array}{l}0 \\
\dot{0} \\
\dot{0}\end{array}} &{\text { 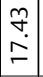 }} &{2 \circ} &{\stackrel{m}{\stackrel{n}{m}}} &{\begin{array}{l}\stackrel{n}{\text { hn }} \\
=\end{array}} &{\text { 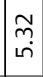 }} &{\begin{array}{l}\text { 苔 } \\
\sigma\end{array}} &{\bar{m}} &{\mid \begin{array}{c}\widehat{\infty} \\
\infty \\
\infty\end{array}} &{\text { 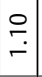 }} &{\text { 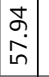 }} &{ } &{ } &{\text { •|lon }} &{\text { 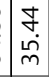 }} &{\circ} &{\mid \begin{array}{l}\frac{1}{2} \\
\hdashline \\
\end{array}} &{\left|\begin{array}{l}q \\
g \\
\infty \\
m\end{array}\right|} &{ } &{\text { ْ }} &{\text { 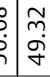 }} &{ } &{\circ^{\circ}} &{\begin{array}{l}\bar{f} \\
n \\
n\end{array}} &{\begin{array}{l}\text { 角 } \\
\multirow{y}{*}{}\end{array}} &{\text { ○० }} &{\begin{array}{c}\tilde{w} \\
\dot{d} \\
\dot{m}\end{array}} &{\text { 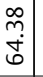 }} &{ } &{ } \\
{\hline} &{=\stackrel{2}{2}} &{\stackrel{2}{9}} &{\stackrel{\infty}{\circ}} &{\text { 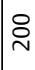 }} &{\text { ฉ }} &{\text { ¿ }} &{\stackrel{\circ}{\frac{1}{N}}} &{z} &{\text { 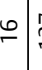 }} &{\hat{m}} &{0} &{\stackrel{m}{=}} &{\text { in }} &{\stackrel{\leftrightarrow}{\circ}} &{m} &{\begin{array}{l}0 \\
0 \\
0\end{array}} &{\bar{\sim}} &{ } &{z \stackrel{\infty}{\gtrless}} &{\tilde{F}} &{z} &{\text { 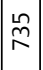 }} &{\text { ơ }} &{\circ=} &{z} &{\text { 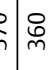 }} &{\hat{\imath}} &{z} &{\frac{O}{\sigma}} &{\begin{array}{l}\stackrel{m}{m}) \\
m\end{array}} &{z} &{\stackrel{\stackrel{\leftrightarrow}{\sim}}{\sim}} &{\begin{array}{l}\stackrel{\partial}{f} \\
\end{array}} &{\begin{array}{l}n \\
\hat{f}\end{array}} &{ } \\
{\hline} &{2} &{\begin{array}{l}\bar{\delta} \\
\dot{D} \\
\dot{v} \\
v\end{array}} &{ } &{ } &{ } &{ } &{ } &{0} &{ } &{ } &{ } &{ } &{ } &{ } &{ } &{ } &{ } &{ } &{\circ \mid \begin{array}{l}\bar{\delta} \\
\dot{D} \\
\dot{v}\end{array}} &{ } &{ } &{\mid \begin{array}{l}\bar{b} \\
\dot{0} \\
\dot{v}\end{array}} &{ } &{ } &{\circ} &{ } &{ } &{ } &{\begin{array}{l}\text { 용 } \\
\text { o. }\end{array}} &{ } &{a} &{\begin{array}{l}\bar{\sigma} \\
\dot{0} \\
\dot{0}\end{array}} &{ } &{ } &{ } \\
{\hline} &{\text { 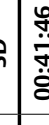 }} &{\begin{array}{l}\infty \\
\stackrel{\leftrightarrow}{\circ} \\
\stackrel{8}{o}\end{array}} &{\begin{array}{|l}\stackrel{0}{0} \\
\dot{\alpha} \\
\ddot{\theta}\end{array}} &{\text { 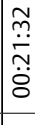 }} &{\begin{array}{l}\infty \\
\ddot{0} \\
\ddot{0} \\
\ddot{\ddot{\theta}}\end{array}} &{\mid \begin{array}{l}0 \\
\hat{\ddot{O}} \\
\ddot{0} \\
\ddot{0}\end{array}} &{\text { 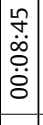 }} &{\text { ८) }} &{\text { 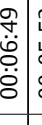 }} &{\text { 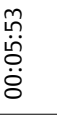 }} &{\mid \begin{array}{l}m \\
\ddot{g} \\
\ddot{\theta}\end{array}} &{\begin{array}{|l}n \\
\stackrel{\ddot{\alpha}}{0} \\
\ddot{\ddot{o}} \\
\end{array}} &{\begin{array}{|l}\stackrel{0}{0} \\
0 \\
\ddot{0} \\
0\end{array}} &{\text { 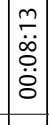 }} &{\text { 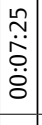 }} &{\text { 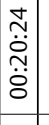 }} &{ } &{ } &{\hat{\imath}} &{\begin{array}{l}\bar{m} \\
\dot{0} \\
\ddot{\theta}\end{array}} &{\text { ฉి }} &{\begin{array}{|l}0 \\
\ddot{\sim} \\
\stackrel{\sim}{0} \\
\ddot{0} \\
\end{array}} &{\mid \begin{array}{l}\stackrel{0}{0} \\
\dot{\alpha} \\
\ddot{\ddot{\theta}}\end{array}} &{ } &{\text { Q }} &{\text { 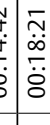 }} &{ } &{\text { जิ }} &{ } &{ } &{\text { î }} &{\text { 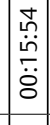 }} &{\text { 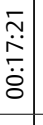 }} &{ } &{ } \\
{\hline} &{\text { 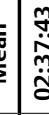 }} &{\text { 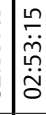 }} &{\text { 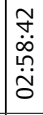 }} &{\begin{array}{l}\text { 足 } \\
\stackrel{\leftrightarrow}{0} \\
\stackrel{\leftrightarrow}{\tilde{o}}\end{array}} &{\text { 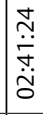 }} &{\text { 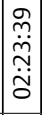 }} &{\text { 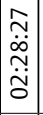 }} &{\text { 苞 }} &{\text { 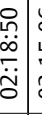 }} &{\text { 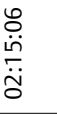 }} &{\text { 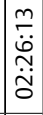 }} &{\begin{array}{|l}\stackrel{m}{\ddot{m}} \\
\stackrel{\vec{q}}{\tilde{d}} \\
\end{array}} &{\text { 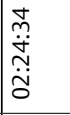 }} &{\text { 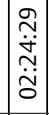 }} &{\text { 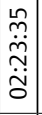 }} &{\text { 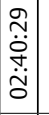 }} &{ } &{ } &{\text { 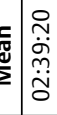 }} &{\text { 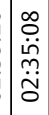 }} &{\begin{array}{l}\mathbf{c} \\
\bar{c} \\
\Sigma \\
\Sigma \\
\end{array}} &{\text { 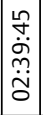 }} &{\mid \begin{array}{c}\bar{n} \\
\dot{\tilde{n}} \\
\dot{\tilde{\sigma}}\end{array}} &{ } &{\text { 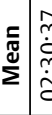 }} &{\text { 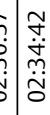 }} &{ } &{\text { 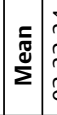 }} &{\text { 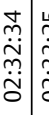 }} &{ } &{\text { 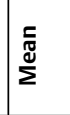 }} &{\mid \begin{array}{c}\hat{\jmath} \\
\dot{\tilde{m}} \\
\dot{\tilde{g}}\end{array}} &{\text { 岕 }} &{ } &{ } \\
{\hline} &{\text { ○ }} &{\begin{array}{l}\stackrel{\bullet}{\circ} \\
\dot{+}\end{array}} &{\underset{\sim}{\tilde{N}}} &{\text { 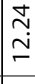 }} &{\begin{array}{l}\bar{\alpha} \\
\dot{\sim}\end{array}} &{\mid \begin{array}{l}\hat{N} \\
\dot{\omega} \\
\sim\end{array}} &{\begin{array}{l}\infty \\
\stackrel{\infty}{2} \\
\stackrel{\sim}{\sim} \\
\end{array}} &{\circ} &{\begin{array}{ll}\infty \\
\infty \\
0 \\
0\end{array}} &{\text { 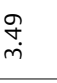 }} &{\text { 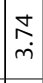 }} &{\stackrel{i}{i}} &{\underset{m}{\stackrel{8}{m}}} &{\text { 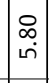 }} &{\begin{array}{l}0 \\
0 \\
0 \\
0\end{array}} &{\begin{array}{l}\circ \\
\stackrel{0}{1} \\
\dot{I}\end{array}} &{ } &{ } &{\stackrel{\bar{\sigma}}{\bar{b}}} &{\text { 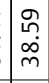 }} &{\circ} &{\text { 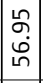 }} &{\mid \begin{array}{l}\tilde{a} \\
\dot{\gamma} \\
\dot{\gamma}\end{array}} &{ } &{\text { × }} &{\text { ר్ }} &{ } &{\circ} &{\text { 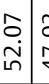 }} &{\text { 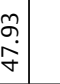 }} &{\text { ○० }} &{\begin{array}{|l|}\infty \\
\stackrel{n}{\sim} \\
m\end{array}} &{\begin{array}{l}\stackrel{\sim}{\checkmark} \\
\mathrm{G}\end{array}} &{ } &{ } \\
{\hline} &{\frac{m}{b}} &{\text { 崫 }} &{\text { 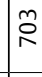 }} &{\tilde{\sigma}} &{\stackrel{\widetilde{N}}{\sim}} &{\text { 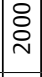 }} &{\frac{8}{2}} &{z} &{6} &{\stackrel{\circ}{\sim}} &{\stackrel{\curvearrowright}{\sim}} &{\text { 占 }} &{\underset{\sim}{\mathbb{N}}} &{\tilde{\tilde{\vartheta}}} &{\text { q }} &{\begin{array}{c}0 \\
0 \\
n \\
n^{n}\end{array}} &{\text { 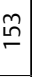 }} &{ } &{\text { 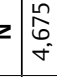 }} &{\text { 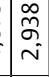 }} &{\mathbf{z}} &{\mid \begin{array}{l}0 \\
m \\
m \\
\dot{f}\end{array}} &{\left|\begin{array}{c}\stackrel{N}{N} \\
m\end{array}\right|} &{\circ 2} &{z \mid} &{\begin{array}{l}\overline{0} \\
\stackrel{\text { N }}{m}\end{array}} &{\text { 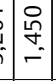 }} &{z} &{\text { 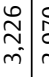 }} &{\text { 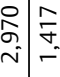 }} &{z} &{\left|\begin{array}{l}0 \\
m \\
\mathcal{N}\end{array}\right|} &{\text { 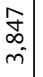 }} &{ } &{\frac{\bar{\sigma}}{\bar{\sigma}}} \\
{\hline} &{2} &{\text { 产 }} &{ } &{ } &{ } &{ } &{ } &{=} &{ } &{ } &{ } &{ } &{ } &{ } &{ } &{ } &{ } &{ } &{\circ \mid \begin{array}{l}\overline{\dot{o}} \\
\dot{v} \\
\dot{v}\end{array}} &{ } &{ } &{\mid \begin{array}{l}\bar{\sigma} \\
\dot{0} \\
\dot{v}\end{array}} &{ } &{ } &{=\mid \begin{array}{l}\bar{a} \\
\vdots \\
\vdots\end{array}} &{ } &{ } &{ } &{ } &{ } &{0} &{\begin{array}{l}\bar{\sigma} \\
\dot{0} \\
\dot{v}\end{array}} &{ } &{ } &{ } \\
{\hline} &{\text { 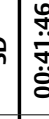 }} &{\text { 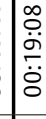 }} &{\begin{array}{|l}\dot{y} \\
\dot{\alpha} \\
\ddot{\theta} \\
\dot{\theta}\end{array}} &{\text { 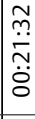 }} &{\text { 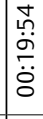 }} &{\text { 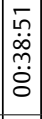 }} &{\text { 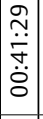 }} &{\text { ฉे }} &{\text { 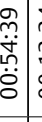 }} &{\begin{array}{l}\stackrel{ \pm}{\ddot{~}} \\
\ddot{8}\end{array}} &{\text { 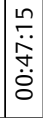 }} &{\begin{array}{l}\overline{\tilde{\tilde{n}}} \\
\ddot{8} \\
\end{array}} &{\text { 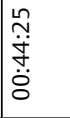 }} &{\mid \begin{array}{c}8 \\
\dot{\tilde{\sigma}} \\
\dot{8} \\
8\end{array}} &{\begin{array}{l}0 \\
\dot{\ddot{q}} \\
\dot{\tilde{g}} \\
\dot{8}\end{array}} &{\text { 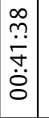 }} &{ } &{ } &{\text { م) }} &{\text { 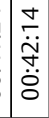 }} &{\text { ถे }} &{\mid \begin{array}{l}0 \\
\ddot{\ddot{\square}} \\
\dot{\square} \\
\end{array}} &{\text { 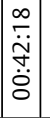 }} &{ } &{\text { น) }} &{\text { 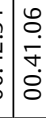 }} &{ } &{\text { ज }} &{\begin{array}{l}\overline{\tilde{j}} \\
\tilde{\tilde{u}} \\
\dot{\Delta}\end{array}} &{\begin{array}{l}0 \\
\stackrel{\square}{\bar{t}} \\
\ddot{8}\end{array}} &{\text { जि }} &{\text { 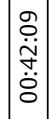 }} &{\text { 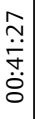 }} &{ } &{ } \\
{\hline} &{\begin{array}{l}\overline{\bar{v}} \\
\bar{\nu} \\
\bar{\nu}\end{array}} &{\text { 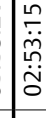 }} &{\begin{array}{|l}\tilde{f} \\
\dot{\infty} \\
\stackrel{\leftrightarrow}{\leftrightarrow ٌ} \\
\tilde{\delta}\end{array}} &{\text { 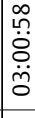 }} &{\text { 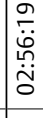 }} &{\begin{array}{|l}\bar{n} \\
\stackrel{\leftrightarrow}{\leftrightarrow} \\
\ddot{\tilde{\theta}} \\
\tilde{0}\end{array}} &{\text { 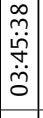 }} &{\text { 敢 }} &{\text { 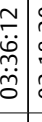 }} &{\text { 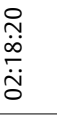 }} &{\begin{array}{l}\stackrel{\sigma}{\ddot{\theta}} \\
\dot{\tilde{\sigma}} \\
\tilde{\sigma}\end{array}} &{\begin{array}{l}0 \\
m \\
\dot{m} \\
\dot{m} \\
\tilde{o}\end{array}} &{\text { 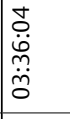 }} &{\begin{array}{l}\tilde{f} \\
\dot{b} \\
\dot{\tilde{g}} \\
\end{array}} &{\begin{array}{c}\tilde{\tilde{O}} \\
\dot{\leftrightarrow} \\
\stackrel{\leftrightarrow}{0} \\
\tilde{O} \\
\end{array}} &{\text { 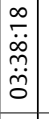 }} &{ } &{ } &{\text { 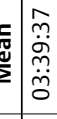 }} &{\text { 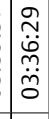 }} &{\text { 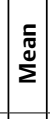 }} &{\mid \begin{array}{c}\hat{n} \\
\hat{\alpha} \\
\tilde{m} \\
\tilde{O} \\
\end{array}} &{\mid \begin{array}{c}\tilde{\ddot{\omega}} \\
\dddot{\tilde{O}} \\
\mid\end{array}} &{ } &{\text { 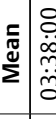 }} &{\text { 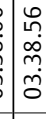 }} &{ } &{\text { 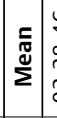 }} &{\text { 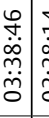 }} &{\text { 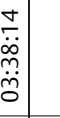 }} &{\mid \begin{array}{l}\bar{\varpi} \\
\sum \\
\sum\end{array}} &{\mid \begin{array}{l}\tilde{\tilde{o}} \\
\dot{\hat{m}} \\
\ddot{\tilde{o}}\end{array}} &{\text { 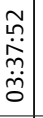 }} &{ } &{\text { 峦 }} \\
{\hline} &{ } &{\stackrel{\circ}{\circ}} &{\frac{9}{0}} &{\text { 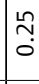 }} &{\stackrel{\stackrel{\sim}{\sim}}{\longrightarrow}} &{\text { 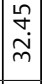 }} &{\text { 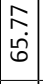 }} &{\circ} &{\frac{-}{\circ}} &{\begin{array}{l}\infty \\
\stackrel{0}{0} \\
0\end{array}} &{\stackrel{\infty}{\stackrel{N}{N}}} &{\begin{array}{|l|} \\
0 \\
0 \\
\dot{0}\end{array}} &{\stackrel{\stackrel{m}{m}}{-}} &{\begin{array}{c}\tilde{c} \\
\stackrel{s}{ } \\
\text { in }\end{array}} &{\text { ְे. }} &{\begin{array}{c}\mathcal{F} \\
\dot{\infty} \\
\infty\end{array}} &{ } &{ } &{\text { : }} &{\text { 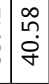 }} &{\circ} &{\begin{array}{|c}m \\
\infty \\
\infty \\
i\end{array}} &{\left|\begin{array}{c}\infty \\
\infty \\
\dot{\bar{\sigma}}\end{array}\right|} &{ } &{\circ} &{\text { 量 }} &{ } &{\circ} &{\text { 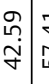 }} &{\begin{array}{c}\bar{\tau} \\
\text { in } \\
\text { in }\end{array}} &{\text { ○ீ }} &{\mid \begin{array}{l}\pi \\
\dot{\sigma} \\
\end{array}} &{\text { ָి }} &{ } &{ } \\
{\hline} &{\text { m }} &{\text { గొ }} &{\tilde{q}} &{\tilde{m}} &{\text { 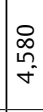 }} &{\mid \begin{array}{l}\tilde{N} \\
\hat{N} \\
\sigma^{\prime} \\
= \\
\end{array}} &{\text { 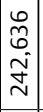 }} &{z} &{\frac{\xi}{\sigma}} &{\text { 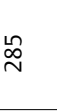 }} &{\text { స్ }} &{\text { 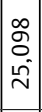 }} &{\text { 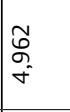 }} &{\text { 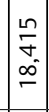 }} &{\bar{q}} &{\begin{array}{|}\bar{m} \\
\bar{b} \\
\bar{m}\end{array}} &{\text { స్. }} &{\text { 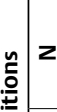 }} &{=\mid \begin{array}{l}\stackrel{\beth}{N} \\
\stackrel{\sigma}{N}\end{array}} &{\mid \begin{array}{l}m \\
\frac{m}{\sigma} \\
g \\
\sigma\end{array}} &{=} &{\text { 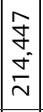 }} &{\text { 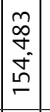 }} &{\circ 2} &{z \mid} &{\begin{array}{l}\circ \\
\stackrel{\infty}{\circ} \\
\stackrel{2}{\circ}\end{array}} &{\text { 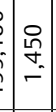 }} &{\text { z }} &{\begin{array}{c}\bar{a} \\
\vdots \\
\vdots \\
\infty \\
\infty\end{array}} &{\text { 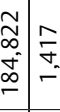 }} &{z} &{\text { 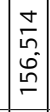 }} &{\text { 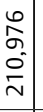 }} &{ } &{\begin{array}{|l}\mathbb{Q} \\
\stackrel{y}{*}\end{array}} \\
{\hline} &{ } &{\text { 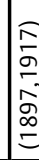 }} &{\text { 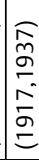 }} &{\text { 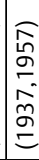 }} &{\text { 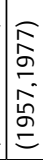 }} &{\text { 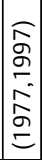 }} &{\text { 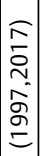 }} &{\text { 密 }} &{\text { 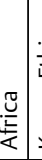 }} &{\text { סِ }} &{1<} &{\text { 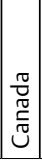 }} &{\text { 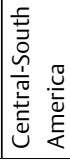 }} &{\text { 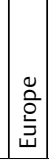 }} &{ } &{\text { | }} &{\text { 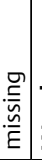 }} &{\text { 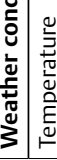 }} &{\text { 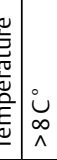 }} &{\mathrm{V}^{\mathrm{v}}} &{\text { 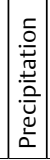 }} &{\text { |ㅎ| }} &{\mid \begin{array}{|c}E \\
E \\
0 \\
\Lambda \\
\Lambda\end{array}} &{\text { 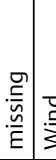 }} &{\stackrel{5}{3}} &{ } &{\text { 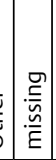 }} &{\text { 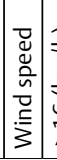 }} &{\begin{array}{l}0 \\
0 \\
0 \\
\wedge\end{array}} &{\text { 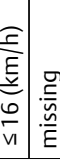 }} &{\text { 辛 }} &{\mid \begin{array}{l}0 \\
\stackrel{0}{1} \\
\wedge\end{array}} &{\begin{array}{l}\stackrel{\circ}{\circ} \\
\stackrel{2}{v_{1}}\end{array}} &{\begin{array}{c}\frac{g}{\bar{\omega}} \\
\frac{\mathscr{E}}{E}\end{array}} &{\text { 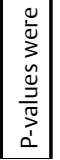 }} \\
$\hline$\end{array}$




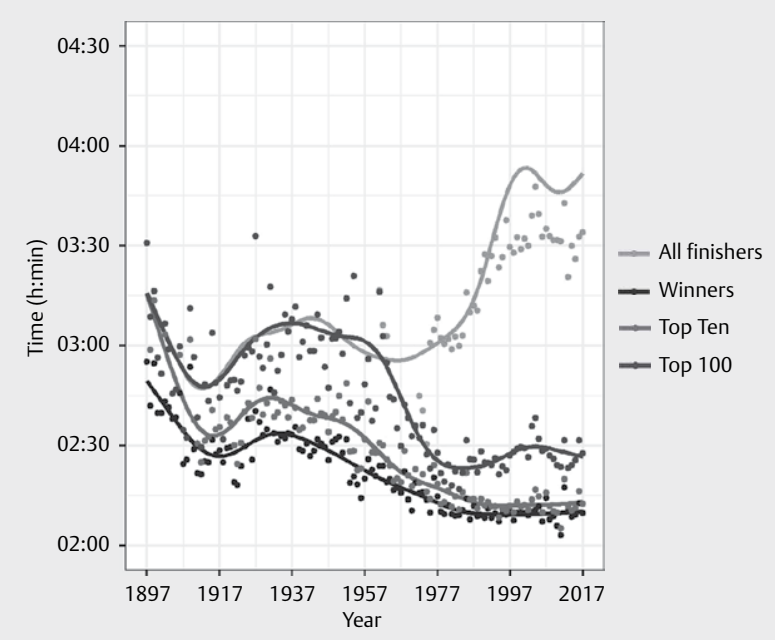

- Fig. 1 Performance of all men finishers, annual hundred fastest (top 100), annual ten fastest (top ten) and winners from 1897 to 2017 , by calendar year. Points are average of time race. Lines are fitted curves. not monotonically, over time. On average, years (1977, 1997) showed the best performances (02:23:39 $\pm 00: 06: 35 \mathrm{~h}: \mathrm{min}: \mathrm{s})$ and the fastest calendar year was 1983 with 02:16:55 $\pm 00: 03: 03 \mathrm{~h}: \mathrm{min}: \mathrm{s}$. Instead, periods $(1937,1957)$ had the worst performance $03: 00: 58 \pm 00: 21: 32$, but 1927 was the slowest calendar year (03:32:54 $\pm 00: 22: 47 \mathrm{~h}: \min : s)$.

Regarding country of origin, athletes from Kenya and Ethiopia were the fastest, 02:15:06 \pm 00:05:53 h:min:s, with a participation of $n=260$ (3.49\%). Athletes from the USA, with the highest participation of $n=5580$ (74.80\%), were the slowest, 02:40:29 \pm 00:20:24h:min:s.

Regarding weather conditions, we did not focus on details, as shown in $>$ Table 1, but we found that temperatures $\leq 8^{\circ} \mathrm{C}$, precipitation $>0 \mathrm{~mm}$, wind direction from the west significantly $(p<0.001)$ improved performance. On the contrary, humidity level $\leq 70 \%$ had a worsening and significant $(p<0.001)$ effect on performance, and also wind speed $\leq 16 \mathrm{~km} / \mathrm{h}$ was not favorable $(p=0.05)$.

\section{Statistical model}

In $>$ Fig. 1 and $>$ Fig. 2 we show the fitted curve, from the generalized additive mixed models, uni-variable and multi-variable, de-
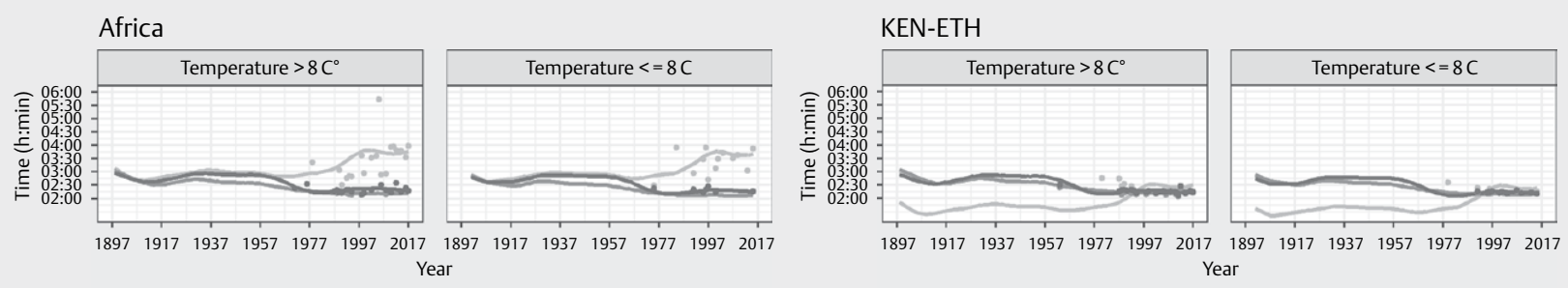
Year
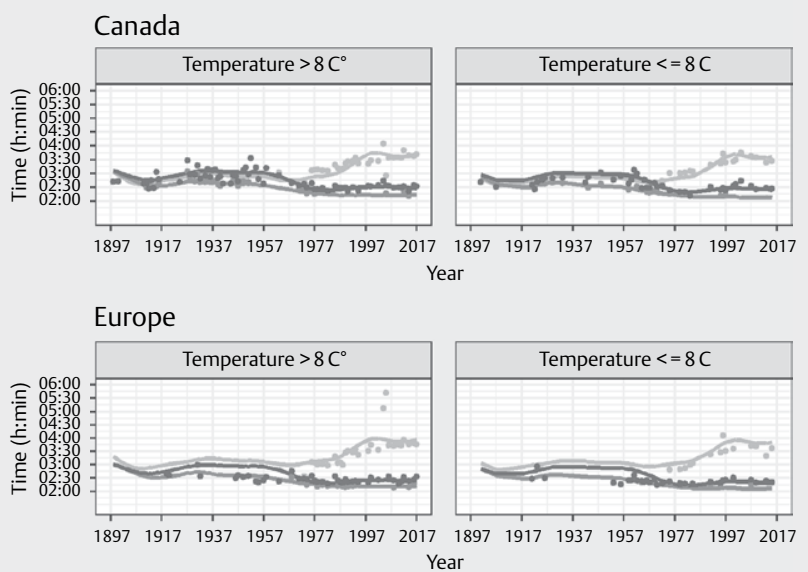

USA

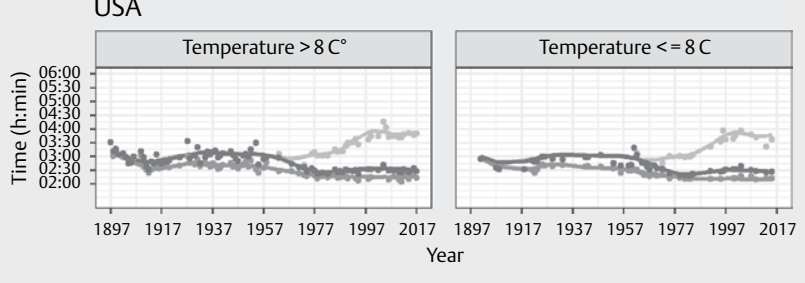

- All finishers $\quad$ Top Ten $\quad$ Top 100

Fig. 2 Performance of all men finishers, annual hundred fastest (top 100) and annual ten fastest (top ten) from 1897 to 2017 , by calendar year, country and air temperature (below or above $8^{\circ} \mathrm{C}$ ). Points are average of time race. Lines are fitted curves. 


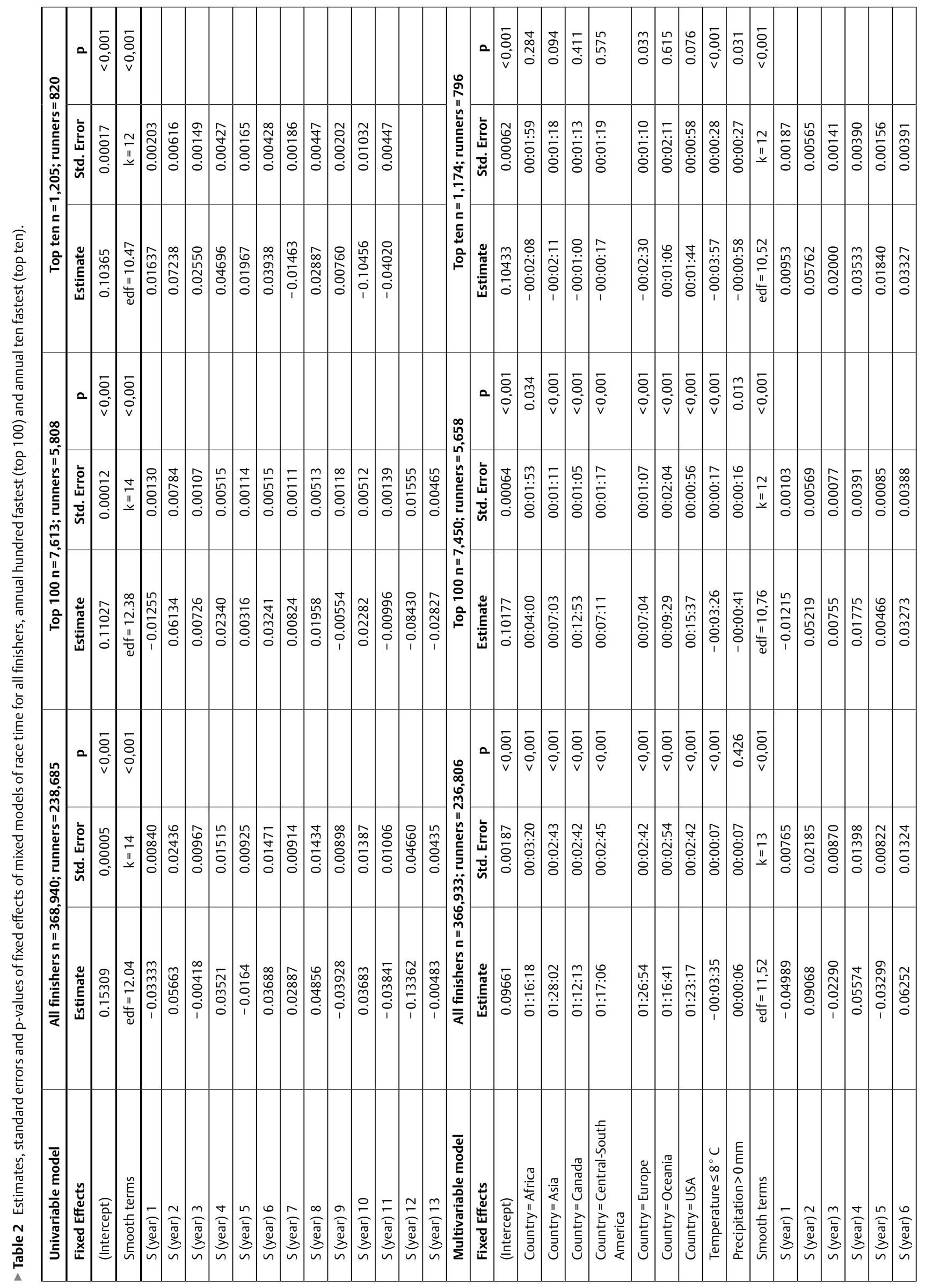




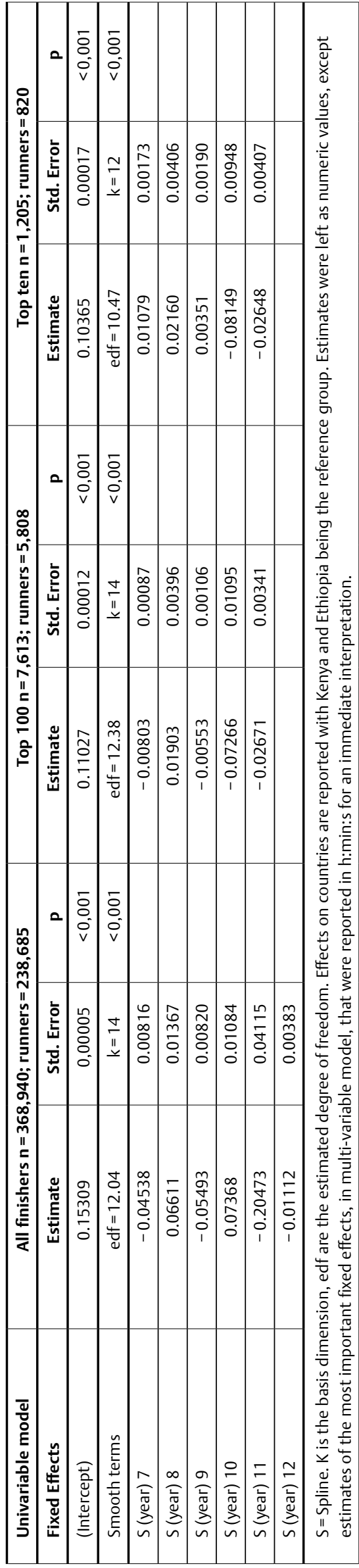

scribed in the methods section and whose details are reported in - Table 2. We do not show, graphically, the effect of precipitation, but only of country and temperature. We observed that race time of the hundred annual fastest finishers decreased across calendar years ( $\triangleright$ Fig. 1 ).

In $\triangleright$ Table $\mathbf{2}$ we show that athletes from Kenya and Ethiopia were significantly the fastest, compared with every other country group. The estimates ranged from a minimum of 00:04:00 h:min:s $(p=0.034)$, which was the estimated difference among the rest of Africa and Kenya-Ethiopia, and a maximum of 00:15:37 h:min:s $(p<0.001)$, which was the estimated difference between USA and Kenya-Ethiopia. Temperature level $\leq 8{ }^{\circ} \mathrm{C}$ significantly $(p<0.001) \mathrm{im}$ proved performance of 00:03:26 h:min:s, compared with temperature $>8{ }^{\circ} \mathrm{C}$. Absence of precipitation was also significant $(p=0.013)$ and improved performance of approximately 00:00:41 h:min:s.

\section{Performance considering the annual ten fastest}

\section{Summary statistics}

A large main effect $(p<0.001)$ of calendar year, grouped into classes, country and weather conditions on race time was shown in - Table 1. In > Fig. 1, we plotted points showing the details of mean performance by calendar year. Performance improved over time. On average, the last period $(1997,2017)$ had the best performances (02:12:23 \pm 00:03:38 h:min:s) and the fastest calendar year was 2011 with 02:05:10 \pm 00:01:29 h:min:s. Instead, the first period $(1897,1917)$ had the worst performance 02:48:09 \pm 00:19:15 h:min:s with 1897 the slowest calendar year (03:30:56 \pm 0:28:56h:min:s).

Regarding the country of origin, Kenya and Ethiopia, the fastest, 02:11:41 \pm 00:03:14 h:min:s, was the second main relevant group in terms of participation, $n=137$ (11.57\%) followed by USA, $n=686$ (57.94\%) which were the slowest, 02:35:07 \pm 00:17:40 h:min:s.

We did not focus on details, as shown in $>$ Table 1 , but we found significantly better performances $(p<0.001)$ on temperature $\leq 8^{\circ}$ $C$ and precipitation $>0 \mathrm{~mm}$. Wind from the west and also humidity level $\leq 70 \%$ were significantly not favorable $(p=0.002$ and $p<0.001$ respectively). Wind speed was not significant $(p=0.154)$.

\section{Statistical model}

In $>$ Fig. $\mathbf{1}$ and $>$ Fig. 2 we show the fitted curve, from the generalized additive mixed models, uni-variable and multi-variable, described in the methods section and whose details are reported in - Table 2. We observed that race time of the ten annual fastest finishers decreased across calendar years ( $>$ Fig. 1). From \ Table 2, athletes from Kenya and Ethiopia seemed not to be the fastest. Significant differences were found (see $>$ Table 2 ) among temperature levels below and above $8{ }^{\circ} \mathrm{C}(p<0.001$, estimated difference $=-00: 03: 57 \mathrm{~h}: \mathrm{min}: \mathrm{s})$. Presence of precipitation was also significant $(p=0.031)$ and improved performance by approximately 00:00:58h:min:s.

\section{Performance considering the annual winners} Summary statistics

A large main effect of calendar year, grouped into classes, on race time was shown $(p<0.001)$ in > Table 1. In > Fig. 1, we plotted points showing the details of mean performance by calendar year. 
Performance improved over time. The first period $(1897,1917)$ had the slowest race time 02:36:09 $\pm 00: 11: 03 \mathrm{~h}: \mathrm{min}: \mathrm{s}$ with 1897 being the slowest calendar year, 02:55:10 h:min:s. On average, the last period $(1997,2017)$ had the fastest race time, 02:09:42 \pm 00:02:55 h:min:s, with 2011 being the fastest calendar year (02:03:02 h:min:s).

In $>$ Table 1, the effect of country on race time seemed significant but in fact it was not relevant, since we did not have, for each country group, enough observations for a good inference. Athletes from Kenya and Ethiopia ( $n=25,20.83 \%$ ) were the fastest with 02:09:20 \pm 00:02:11 h:min:s and athletes from Canada $(n=15$, $12.50 \%$ ) were the slowest with 02:30:48 \pm 00:06:56 h:min:s.

We found significantly better performances at temperatures below $8{ }^{\circ} \mathrm{C}(02: 18: 15 \pm 00: 08: 36 \mathrm{~h}: \min : s$ versus 02:24:12 \pm 00:13:01 h:min:s, $\mathrm{p}=0.003)$, and precipitation > $0 \mathrm{~mm}(02: 19: 03 \pm$ 00:10:31 h:min:s versus 02:23:31 $\pm 00: 11: 58 \mathrm{~h}: \min : \mathrm{s}, \mathrm{p}=0.034)$. Other weather conditions were not significant.

\section{Statistical model}

In $>$ Fig. 1 we show the fitted curve, from the uni-variable generalized additive mixed model described in the methods section but whose details were not reported in $>$ Table 2 . Race time of the annual winners decreased across calendar years. Since we did not have enough observations, we did not perform a multivariable analysis as we did for all the finishers and annual top ten finishers.

\section{Discussion}

We investigated participation and performance trends in male runners competing in the Boston Marathon since the first race held in 1897. We performed both descriptive and inferential statistics. By analyzing all annual finishers, the annual hundred, ten fastest and the annual winners, the main findings were (i) the number of finishers increased dramatically from the middle of 1970s, (ii) the fastest race times were achieved in the beginning of the race when all annual finishers were considered, (iii) the fastest race times were achieved in the first two decades of the actual century when the annual ten fastest and annual top runners were considered and (iv) Ethiopian and Kenyan runners were the fastest (v) air temperature (i.e. cold temperatures) and precipitation had a significant effect on race performance.

\section{Performance considering all finishers}

When all finishers were considered since the beginning of the race, the number of finishers increased dramatically from the middle of 1970 s. This increase in the seventies is most likely due to the increase in age group runners $[8,12]$ and the 'running boom' that hit the United States from the late 1970 s onward $[2,19]$. For example, between 1968 and 1976 the number of marathons increased in the United States of America by 300 \% [14]. We see in 1996 a very high number of finishers. In that year, the historic $100^{\text {th }}$ event of the Boston Marathon attracted 38,708 entrants with 36,748 starters and had 35,868 official finishers, which stood as the largest field of finishers in the history until 2004 (www.baa.org/races/boston-marathon/boston-marathon-history/boston-marathon-milestones. aspx). Although we examined here the trends in participation and performance for men in the Boston Marathon, future studies also need to investigate the trends in participation in women in large city marathons such as the New York City Marathon.

Furthermore, race times of all finishers increased across calendar years in line with participation. This is most likely due to the increased variability on performance introduced by the increased number of age group runners $[8,12]$. Although these age group runners improved their race times in the last decades, they still run considerably slower compared to the elite marathoners. Therefore it seems that the effect of increasing participation resulting with worsening race times is greater than the improvement in performance with time passing and with improvement conditions.

The first period of observation between 1897 and 1917 had the fastest race times, with the overall best performance in 1912. During the last 20 years, the slowest race times were found with the year 2004 having the overall slowest race time. This is again most likely explained by the increase in participation of age group runners in marathon races $[8,12]$. When running times and age of all 415,000 runners in the New York City Marathon from 1983 to 1999 were examined, the number of master participants increased at a greater rate than their younger counterparts [8].

\section{Performance considering elite athletes (annual ten fastest and annual winners)}

Differences in performance trends were, however, found for elite runners. When the annual ten fastest were considered, the best performance were achieved in the last 20 years, whereas 2011 was the fastest year. However, the slowest race times were found in the first year $(1897)$ and the first period $(1897,1917)$ of the race. We also observed that race times of the ten annual fastest finishers decreased across calendar years. The same results were also obtained when the annual winners were considered.

In the beginning of the Boston Marathon, the early men had to run on muddy roads, with bad shoes, bad nutrition, no fluids, and little training (personal communication Amby Burfoot, winner Boston Marathon in 1968). Now, training and pre-race preparation have changed and the elite East African runners are professional athletes. For elite athletes, the trend of improvement is still going on. When running data from 150 years of sprinting and distance running were analyzed, performance improved annually towards an asymptotic limit [26].

\section{Participation and performance of East African runners}

An important finding was that runners from East Africa showed the smallest participation but the fastest race times. It is well-known that East African runners from Ethiopia and Kenya are the fastest in marathon running when taking into consideration the fastest race times in marathon running from the world best list of the International Association of Athletics Federations (IAAF) [17] and the races of the World Marathon Majors [9].

What is new and very astonishing is the finding that the average race times decreased in Kenyan and Ethiopian runners since they entered the race in 1979 and 1963, respectively. For all other countries, race times increased. This might be explained by the different motivation in East-African runners compared to runners from other countries. It is well-known that runners from Kenya [18] and Ethiopia [21] are motivated to run large city marathons in order to win prize money. However, also superior physiological capacity is 
necessary in East-African runners to compete faster than runners from another origin [11].

\section{The influence of environmental conditions}

We showed p-values and summary statistics of environmental conditions such as air temperature, wind direction, wind speed, precipitation and humidity on race day. Race times were faster at low temperatures $\left(\leq 8{ }^{\circ} \mathrm{C}\right.$ ), with precipitation, humidity level $\leq 70 \%$, wind from West and wind speed $\leq 16 \mathrm{~km} / \mathrm{h}$, depending on whether all finishers, the annual 100 fastest, the annual 10 fastest or the annual winners were considered. However, for the annual winners, wind from west, humidity and wind speed showed no influence on race times.

The influence of weather on race times in marathon running is well-known [3-6, 16, 24, 25]. It has been reported that warmer temperatures impair performance $[4,15,16]$, especially in slower runners $[3,5,6,16]$. The influence of weather on race times has already been investigated for the Boston Marathon [13, 15, 20, 23]. However, it has been investigated only in limited samples such as the top 3 for 30 years [23], winning times for 1933-2004 [15] or the top 10 male and 10 female finishers from 2005 to 2014 [13].

Our study provides now, however, more and detailed data for the whole period 1897-2017 and for different performance levels of male runners. An interesting finding was that temperature $\leq 8^{\circ} \mathrm{C}$, precipitation, wind from west, wind speed $\leq 16 \mathrm{~km} / \mathrm{h}$ and humidity level $\leq 70 \%$ improved race times when considering all finishers. We can confirm previous findings of Trapasso et al. [23]. These authors reported that record-breaking performances were characterized by a wet bulb temperature of less than $7.8{ }^{\circ} \mathrm{C}$ and $100 \%$ sky cover where a light drizzle was also conducive to better performance.

However, for the annual 10 fastest and the annual winners, wind speed showed no influence on performance. A potential explanation for this finding could be the running speed of the athletes. While the top runners compete at a running speed of $\sim 20 \mathrm{~km} / \mathrm{h}$, they might compensate for the influence of wind. And a potential explanation for the improved performance a low temperatures and with precipitation could be 'external cooling' [22].

Our statistical model confirmed that air temperature (i.e. cold temperatures) significantly improved performance for all finishers, the annual hundred and ten fastest. In addition, precipitation significantly improved race performance for the annual hundred and ten fastest.

\section{Limitations, strength and practical application}

The findings of the present study are limited by the specific characteristics of the race in terms of participation and qualifying criteria. Thus, they should be generalized with caution to other marathon races. Moreover, we did not have information about age, and we could not exactly identify repeated measurements within runners although we could reasonably suppose that two observations belonged to the same runner, if they had in common both name, surname, country and participation, once a year, in the same period of time.

A further limitation is that we must be aware that not all official finishers in the early years were recorded when comparing the number of finishers with the number of participants (www.bostonmarathonmediaguide.com/qualification/participation/). In fact it seems unusual that, considering all finishers, the year 1912 was the fastest calendar year (02:29:31 \pm 00:04:27 h:min:s). According to the race director, "The only results we have are those recorded by the athletics officials, who often went home before the last finishers completed the course" (personal communication). In later years, the number of finishers corresponds well with the number of participants. We also have to mention that the Boston Marathon is the only large city marathon in the world with qualifying times (www.baa.org/races/boston-marathon/participant-information/ qualifying/history-of-qualifying-standards.aspx) and the qualifying standards could favor one sex and/or age group over the other.

Although qualification criteria exist, around 10,000 runners participate on sponsor loyalties. Most likely, the number of this latter group of runners has increased most over the year, while qualifiers remained stable over the years. It can be expected that the qualified age-groupers will perform better than their free-entry counterparts; however, as this information is unavailable, this speculative hypothesis cannot be tested critically.

Unfortunately, we did not have the complete list of all runners belonging to push rim wheelchair division starting in 1975. We attempted to exclude this category by eliminating runners with race times shorter than the annual top record.

We also should be aware that in the first 27 years the distance was not standardized at $42.195 \mathrm{~km}$ (26 miles, 385 yards). From 1897 to 1923 , the race distance was $\sim 37 \mathrm{~km}$, from 1924 to 1926 at $\sim 40 \mathrm{~km}$, since 1927 exactly $42.2 \mathrm{~km}$, and since 1957 the official distance of $42.195 \mathrm{~km}$ (www.baa.org/races/boston-marathon/boston-marathon-history.aspx). Therefore, results based on data before 1927 must be interpreted with caution.

Another bias could arise from incomplete information on the year 2005, which was integrated with data coming from www.marathonguide.com/results. In fact, for this year, information about nationality might not be exact or reliable, since it came from a different source.

Considering all observations, the large amount of data and the large number of runners (clusters) made inferential statistics computationally challenging and obtaining a model with a better fit was not feasible. In particular, > Fig. 1, the uni-variable model fit for all finishers looked weaker, compared to the other runners groups, especially in the last 20 years. However, the fit in the multi-variable model looked better ( $>$ Fig. 2). Moreover, comparing performance by countries over the whole period of observation was an easy way to identify different patterns in different countries ( Fig. 2), but it could not be an accurate measure of country effect on performance, first of all, because we compared countries that participated several years before Kenya and Ethiopia, the reference group, entered for the first time. This fact caused the extremely low fit of performance by Kenya and Ethiopia before the eighties, considering all finishers, and it also might have affected the results in the multi-variable model for the annual ten fastest, where it seemed that Kenya and Ethiopia were not the fastest.

In the environmental analysis, both temperature and participation were added as categorical variables into the model, based on previous research. We did not show, but we verified, that including these variables as continuous variables would not much improve the model fit, though the effects of weather conditions should have 
been more accurate, but this was beyond the main goals of this study and would be the subject of further research.

So our results should be interpreted with caution due to the limited information available. On the other hand, the strength of the study was that it analyzed trends in performance and participation in one of the most popular marathon races worldwide, considering all finishers ( > half a million) through its history. Since marathon running continues to increase its popularity, the findings are of great interest for coaches and trainers working with marathon runners, as well as for scientists focusing on human performance.

\section{Conclusions}

In summary, participation increased dramatically in the Boston Marathon from the middle of 1970s and this perhaps worsened performance because race times significantly increased in time. However, when the annual ten fastest and the annual winners were considered, performance improved over time and race times decreased. When the annual hundred fastest are considered, performance improved with time but not monotonically. We found significant differences among runners from East Africa and other countries for all finishers and the annual hundred fastest. We also found significant effects of weather conditions on performance. Race times were faster at low temperatures $\left(\leq 8^{\circ} \mathrm{C}\right)$, for all finishers, the annual 100 and 10 fastest, and with precipitation, for the annual 100 and 10 fastest. This would require further research. These findings need to be confirmed in other large city marathons such as the New York City Marathon. Future studies also need to investigate the influence of weather on different performance levels in both female and male runners.

\section{Acknowledgments}

The help of Amby Burfoot through his constructive criticism and comments is gratefully acknowledged.

\section{Conflict of Interest}

No conflict of interest has been declared by the authors.

\section{References}

[1] Ahmadyar B, Rosemann T, Rust CA, Knechtle B. Improved race times in marathoners older than 75 years in the last 25 years in the world's largest marathons. Chin J Physiol 2016; 59: 139-147

[2] Burfoot A. The history of the marathon: 1976-present. Sports Med 2007; 37: 284-287

[3] El Helou N, Tafflet M, Berthelot G, Tolaini ], Marc A, Guillaume M, Hausswirth C, Toussaint JF. Impact of environmental parameters on marathon running performance. PloS One 2012; 7: e37407

[4] Ely MR, Cheuvront SN, Montain SJ. Neither cloud cover nor low solar loads are associated with fast marathon performance. Med Sci Sports Exerc 2007; 39: 2029-2035

[5] Ely MR, Cheuvront SN, Roberts WO, Montain SJ. Impact of weather on marathon-running performance. Med Sci Sports Exerc 2007; 39: 487-493
[6] Ely MR, Martin DE, Cheuvront SN, Montain SJ. Effect of ambient temperature on marathon pacing is dependent on runner ability. Med Sci Sports Exerc 2008; 40: 1675-1680

[7] Harriss DJ, Macsween A, Atkinson G. Standards for ethics in sport and exercise science research: 2018 update. Int J Sports Med 2017; 38: 1126-1131

[8] JokI P, Sethi PM, Cooper AJ. Master's performance in the New York City Marathon 1983-1999. Br J Sports Med 2004; 38: 408-412

[9] Knechtle B, Aschmann A, Onywera V, Nikolaidis PT, Rosemann T, Rust CA. Performance and age of African and non-African runners in World Marathon Majors races 2000-2014. J Sports Sci 2017; 35: 1012-1024

[10] Kuscsik N. The history of women's participation in the marathon. Ann N Y Acad Sci 1977; 301: 862-876

[11] Larsen HB, Sheel AW. The Kenyan runners. Scand J Med Sci Sports 2015; 25: 110-118

[12] Lepers R, Cattagni T. Do older athletes reach limits in their performance during marathon running? Age 2012; 34: 773-781

[13] Maffetone PB, Malcata R, Rivera I, Laursen PB. The Boston Marathon versus the World Marathon Majors. PloS One 2017; 12: e0184024

[14] Maron MB, Horvath SM. The marathon: A history and review of the literature. Med Sci Sports 1978; 10: 137-150

[15] Miller-Rushing AJ, Primack RB, Phillips N, Kaufmann RK. Effects of warming temperatures on winning times in the Boston marathon. PloS One 2012; 7: e43579

[16] Montain SJ, Ely MR, Cheuvront SN. Marathon performance in thermally stressing conditions. Sports Med 2007; 37: 320-323

[17] Nikolaidis PT, Onywera VO, Knechtle B. Running performance, nationality, sex, and age in the $10-\mathrm{km}$, half-Marathon, marathon, and the 100-km ultramarathon IAAF 1999-2015. J Strength Cond Res 2017; 31: 2189-2207

[18] Onywera VO, Scott RA, Boit MK, Pitsiladis YP. Demographic characteristics of elite Kenyan endurance runners. J Sports Sci 2006; 24 : 415-422

[19] Pate RR, O'Neill JR. American women in the marathon. Sports Med 2007; 37: 294-298

[20] Roberts WO. Heat and cold: What does the environment do to marathon injury? Sports Med 2007; 37: 400-403

[21] Scott RA, Georgiades E, Wilson RH, Goodwin WH, Wolde B, Pitsiladis YP. Demographic characteristics of elite Ethiopian endurance runners. Med Sci Sports Exerc 2003; 35: 1727-1732

[22] Stevens C], Kittel A, Sculley DV, Callister R, Taylor L, Dascombe B]. Running performance in the heat is improved by similar magnitude with pre-exercise cold-water immersion and mid-exercise facial water spray. J Sports Sci 2017; 35: 798-805

[23] Trapasso LM, Cooper JD. Record performances at the Boston Marathon: Biometeorological factors. Int J Biometeorol 1989; 33: 233-237

[24] Trubee NW, Vanderburgh PM, Diestelkamp WS, Jackson KJ. Effects of heat stress and sex on pacing in marathon runners. J Strength Cond Res 2014; 28: 1673-1678

[25] Vihma T. Effects of weather on the performance of marathon runners. Int J Biometeorol 2010; 54: 297-306

[26] Weiss M, Newman A, Whitmore C, Weiss S. One hundred and fifty years of sprint and distance running - Past trends and future prospects. Eur J Sport Sci 2016; 16: 393-401

[27] Zavorsky GS, Tomko KA, Smoliga JM. Declines in marathon performance: Sex differences in elite and recreational athletes. PloS One 2017; 12: e0172121 


\section{Supplementary Material}

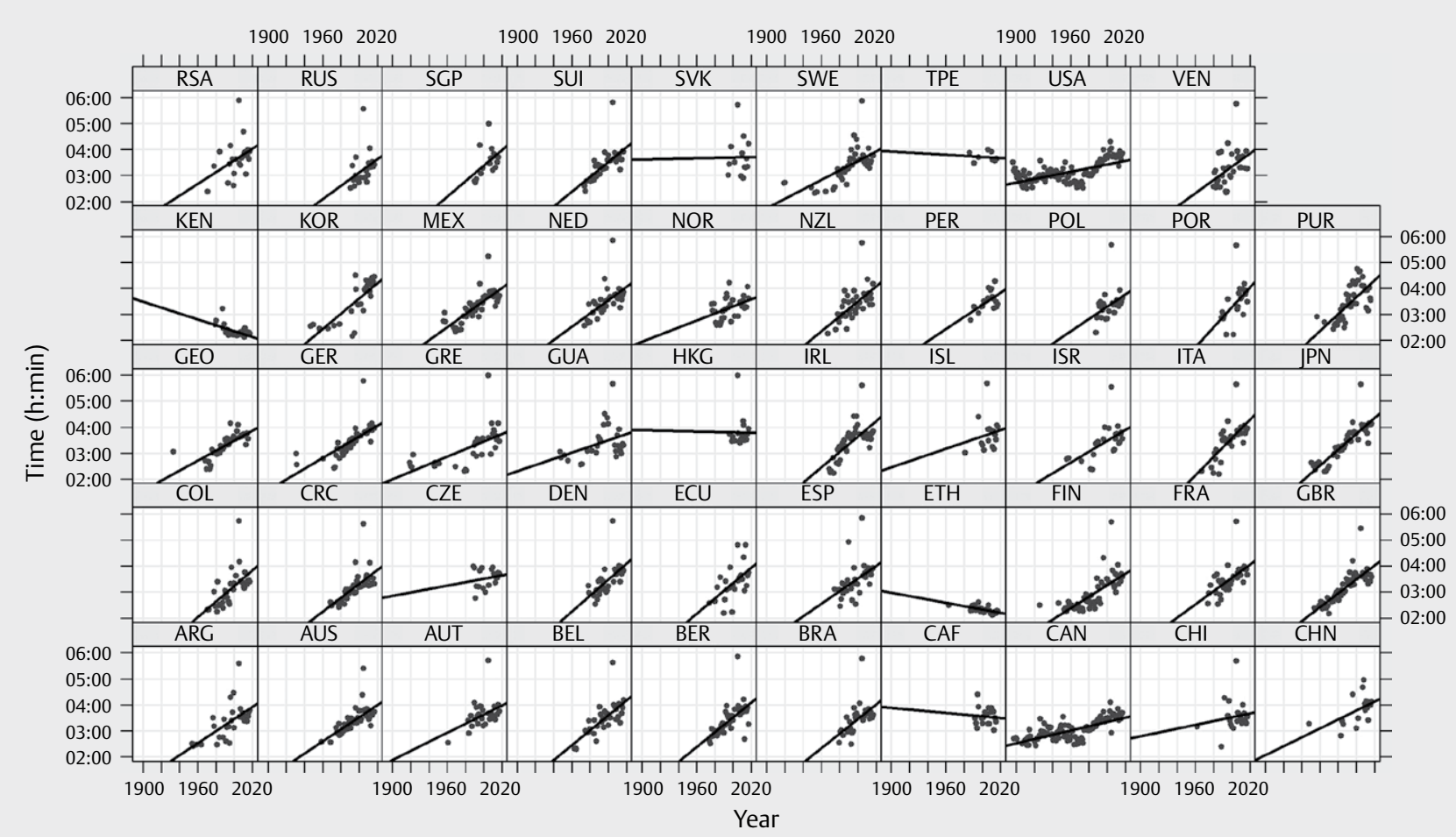

- Fig 15 Performance of all men finishers, for the most relevant nationalities, denoted with IOC codes, from 1897 to 2017 , by calendar year. Points are average of time race. Lines are the linear trend. 